\title{
La universidad entre lo real y lo virtual: una trayectoria no lineal para la didáctica universitaria
}

\author{
Jesús Salinas ${ }^{1}$ \\ Universitat de les Illes Balears \\ Victoria I. Marín ${ }^{2}$ \\ Universitat de les Illes Balears
}

\begin{abstract}
Resumen: El Espacio Europeo de Educación Superior (EEES) ha implicado cambios sustanciales en el aspecto de lo virtual en la didáctica universitaria. La introducción de entornos virtuales de enseñanzaaprendizaje permite y requiere de un cambio en el enfoque de la didáctica universitaria, en relación a los roles que desempeñan profesorado y alumnado. Estos entornos, si bien en un inicio podían ser únicamente un reflejo de la clase tradicional, cada vez más se orientan a la permeabilidad de contextos y situaciones didácticas, hasta el punto de que a veces es difícil establecer las fronteras entre la virtualidad y la realidad, así como el ámbito formal e informal. Las posibilidades que ofrecen los entornos virtuales de aprendizaje se orientan al aprendizaje ubicuo, en cualquier momento y lugar, y por tanto, a lo largo de la vida. Por ello, la integración de estos entornos en la didáctica universitaria suponen un cambio necesario en las metodologías de enseñanza-aprendizaje a favor del aprendiz y requieren, por otro lado, una adecuada formación del profesorado.
\end{abstract}

Palabras clave: didáctica universitaria, aprendizaje ubicuo, virtualidad, metodologías activas, entornos de aprendizaje.

Abstract: The European Higher Education Area (EHEA) has led to substantial changes in the appearance of the virtual in university teaching. The introduction of virtual teaching-learning allows and requires a change in approaching to university teaching, especially related to the roles of teachers and students. These environments, although initially may only be a reflected version of the traditional classroom, now are increasingly oriented to the permeability of teaching contexts and situations. Sometimes it is even difficult to establish the boundaries between reality and virtuality and the formal and informal level. The potential of virtual learning environments are geared towards ubiquitous learning; in other words: anytime, anywhere, and therefore throughout life. Thus, the integration of these environments in university teaching must involve a change in the teaching-learning methodologies for learners and requires, on the other hand, suitable teacher training.

Keywords: university didactics, ubiquitous learning, virtuality, active methodologies, learning environments.

\section{Introducción}

Entre los cambios que se han ido produciendo en la educación superior por la incorporación de los procesos de enseñanza-aprendizaje a los entornos virtuales, está

\footnotetext{
${ }^{1}$ Investigador principal del Grupo de Tecnología Educativa de la Universitat de les Illes Balears (UIB, España). Doctor en Filosofía y Ciencias de la Educación, Licenciado en Filosofía y Letras (Sec. CC Educación) y Diplomado en Profesorado de EGB. Docente de Grado, Postgrado y Doctorado. Director del Institut de Recerca i Innovació Educativa de la UIB.

${ }^{2}$ Investigadora del Grupo de Tecnología Educativa de la Universitat de les Illes Balears (UIB, España). Doctora en Tecnología Educativa. Profesora asociada del área de Tecnología Educativa en el Departamento de Pedagogía Aplicada y Psicología de la Educación de la UIB.
} 
sin duda una creciente preocupación por temas didácticos. De esta manera, podemos entender que la preocupación por la didáctica universitaria está muy ligada al desarrollo de lo virtual, fundamentalmente porque la traslación de los procesos formativos a entornos virtuales no resiste bien los modelos convencionales y esto nos lleva a preocupaciones por nuevas metodologías, por el aprendizaje del alumno, etc.

Lo que se espera hoy en día de la Didáctica universitaria es que nos ayude a conocer mejor lo que sucede en los procesos de enseñanza y aprendizaje que se producen en la Educación Superior, tal como señala Zabalza (2008, p.494), "Ello nos posibilitará crear y sistematizar un cuerpo de conocimientos y de metodologías capaces de incidir en la mejora de las prácticas docentes; nos ofrecerá herramientas conceptuales y operativas para generar estrategias de acción capaces de mejorar cualitativamente los procesos de enseñanza-aprendizaje que llevamos a cabo en nuestras clases o a través de mediaciones virtuales".

\section{¿Qué entendemos por Didáctica Universitaria?}

No se dispone de una definición comúnmente aceptada de qué significa didáctica universitaria. Para Zabalza (2008, p.493), "la Didáctica actual es ese campo de conocimientos, de investigaciones, de propuestas teóricas y prácticas que se centran en los procesos de enseñanza y aprendizaje: cómo estudiarlos, cómo llevarlos a la práctica en buenas condiciones, cómo mejorar todo el proceso".

Se espera entonces que proporcione fundamentos conceptuales y metodológicos que orienten la comprensión del fenómeno de la enseñanza y el fortalecimiento del ejercicio docente como tal. Y que, en el tema que nos concierne, contribuya a generar procesos de enseñanza-aprendizaje que utilicen adecuadamente los instrumentos que proporcionan los Entornos Virtuales de Enseñanza-Aprendizaje (EVEA).

La Didáctica universitaria se refiere, en principio, a todo el profesorado, sea cual sea su especialidad o la materia que enseña: "Todos los profesores y profesoras universitarias son didactas. Todos ellos y ellas enseñan y deben poseer las dotes (las competencias) que les permitan llevar a cabo esa tarea: el enseñar con eficacia" (Zabalza, 2011, p.391).

La docencia - función inherente a la universidad- es considerada como un proceso organizado, intencional y sistemático que orienta los procesos de enseñanzaaprendizaje, exigiendo en el profesor el dominio de su especialidad, así como el conocimiento de las estrategias, técnicas y recursos psico-pedagógicos que promuevan la interacción didáctica y los aprendizajes significativos en los usuarios. Entre las habilidades didácticas necesarias para ello, pueden señalarse (Zabalza, 2008, 2011; Batista, Borges y Forés, 2006):

- habilidad esencial para enseñar

- habilidad de comunicación

- habilidad de organización

- habilidad de retroalimentación

- $\quad$ habilidad de monitoreo

- habilidad de revisión y cierre 
Si ha habido un hito determinante en la evolución de la preocupación didáctica asociada a lo virtual, al menos nominalmente, este ha sido la implantación del Espacio Europeo de Educación Superior (EEES). Es en el terreno de lo virtual donde encontramos mayores impactos del EEES en relación a la didáctica universitaria, sobre todo porque permiten la gestión por parte de los docentes de actividades individualizadas y la incorporación tímida de cambio de metodologías, de orientación al alumno, etc.

Junto a ello, la incorporación de parte de las acciones formativas a los EVEA requiere un cambio de rol por ambas partes, alumnos y profesores. Cambios que implican forzosamente nuevas formas de docencia, pasando de procesos meramente transmisivos a procesos donde el alumno dispone de autonomía y cierto control sobre el proceso.

Uno de los aspectos más importantes que hace referencia a la intersección de la didáctica con los EVEA tiene que ver con la capacitación y actualización del profesorado para incorporar las TIC en su práctica. Sea cual sea la modalidad (cursos y talleres convencionales, comunidades de intercambio de experiencias, redes de expertos, desarrollo de buenas prácticas, gestión de un portafolios profesional, etc.), lo importante son las competencias digitales en la docencia de forma integrada, con tipos de conocimientos relativos al dominio del contenido a enseñar y al dominio de recursos y estrategias didácticas adecuadas (Salinas, de Benito \& Lizana, 2014).

Este tipo de conocimiento integrado puede abordarse muy bien desde el modelo TPACK (Technological, Pedagogical and Content Knowledge) que implica utilizar una metodología efectiva para el uso de las TIC apoyando estrategias y métodos pedagógicos en relación a una disciplina concreta. El modelo TPACK surge para identificar los conocimientos requeridos para integrar las TIC en la docencia (Shulman, 1986), a los que Mishra y Koehler (2008) añadieron el conocimiento tecnológico a los dos tipos de conocimientos -pedagógico y del contenido-, propuestos por Shulman. Engloba tres tipos de conocimientos: conocimiento del contenido, conocimiento tecnológico y conocimiento pedagógico que, entremezclados, dan lugar a siete tipos de conocimientos (Fig.1). Este modelo se ajusta perfectamente a lo que acontece en la docencia universitaria.

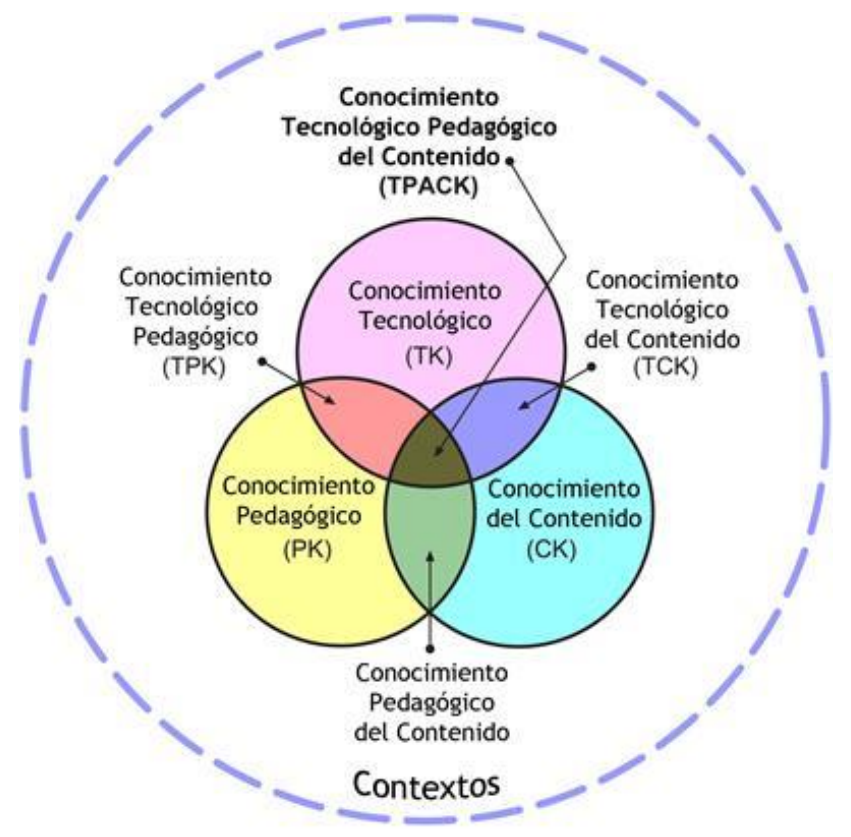

Figura 1. Modelo TPACK. Reproducido con el permiso del editor, ( 2012 por tpack.org. 


\section{La incorporación de lo virtual en las instituciones de educación superior}

Puede decirse que el proceso de virtualización en las universidades se inicia en la mitad de la década de los 90, cuando comienzan a incorporar una oferta a distancia o semipresencial (o blended learning) basada en lo que en ese momento eran los LMS incipientes (o las soluciones diseñadas ad-hoc) para constituir lo que entendemos por EVEA.

Anteriormente, podemos encontrar experiencias que proyectan en ambientes de formación lo que ya se venía utilizando en el ámbito de los juegos: los MUD (Multi-User Dungeon) vienen a ser mundos virtuales multi-jugador en tiempo real basados en texto -al menos en un primer momento- y que a mediados de los 90 comienzan a incorporar gráficos cada vez de mayor resolución; o los MOO (MUD orientados a objetos), sistemas accesibles vía red, multi-usuario, programables e interactivos muy adecuados para la construcción de los juegos de aventura basados en texto, sistemas de conferencias y otros software de colaboración. Un ejemplo de su proyección al ámbito educativo puede ser First-class utilizado a partir de 1989.

Con el siglo XXI, las soluciones de virtualización de elementos del proceso de enseñanza-aprendizaje van generalizando proyectos y servicios de apoyo, a la vez que concentrándose y evolucionando los LMS utilizados. En esta época van consolidándose distinto tipo de entornos virtuales como escenarios donde se desarrolla la formación superior. La implantación del EEES resulta un espaldarazo para el crecimiento de los campus virtuales y de todo tipo de soluciones de virtualización.

En este contexto, la oferta de las universidades se ha diversificado y amplificado, orientándose cada vez más hacia la virtualidad y su consecuente flexibilidad en el aprendizaje. Este aprendizaje flexible implica (Salinas, 2002):

- libertad del alumno en cuanto al lugar, tiempo, métodos y ritmo de enseñanza y aprendizaje

- énfasis en el aprendiz en vez del profesor

- cambio de rol del profesor a mentor y facilitador del aprendizaje

- ayudar a los alumnos a ser autónomos en su aprendizaje a lo largo de toda la vida

En este proceso de incorporación de lo virtual a la educación superior los EVEA suponen una primera aproximación a virtualizar el ámbito o escenario de aprendizaje. Mediante distintas metáforas se trasladan al mundo digital elementos y figuras como el aula, la lección, la exposición y entrega del contenido, la discusión y otros elementos clave del proceso de enseñanza-aprendizaje.

Junto a esta virtualización del escenario, evolucionan otros elementos de que lo complementan, haciendo avanzar el proceso: la posibilidad de una identidad digital que virtualiza los elementos de comunicación del sujeto, sobre todo a través de redes sociales, etc; la utilización de avatares de distinto tipo, y el desarrollo de espacios en 3D donde estos evolucionan. Todo ello propicia una rápida evolución de conceptos como e-learning, blended learning, etc, que hacen referencia, sobre todo, a la virtualización de actividad del alumno en estos entornos. 
La permeabilidad entre lo real y lo virtual, las nuevas formas de virtualidad (nuevos escenarios)

Los avances en la transmisión de datos (cantidad, calidad, seguridad,...) y la digitalización, junto a los avances en el entorno del usuario (interacción personamáquina, etc.) multiplican las posibilidades de la virtualización a través de la red. Aquí el verbo más apropiado para describir el modo de interacción persona-máquina no es 'conversar' sino 'explorar'. Este simple cambio de enfoque tiene una gran diferencia para el resto de fenómenos que se dan en las redes (desde los procesos de diseño de materiales, a los interfaces, los modos de distribución, etc.) al transformarse en un ámbito para la telepresencia, de modo que tengamos la impresión de haber sido transportados (cuerpo incluido) del mundo físico habitual al virtual, a los mundos de la imaginación en el sentido de la extensión de los sentidos de MacLuhan. Además de acceso a intercambio de información, ahora nos proporciona el acceso a nuevas percepciones.

Por tanto, se trata de nuevos escenarios de aprendizaje que apuntan hacia formas de virtualidad en las que no siempre está clara la frontera entre lo real y lo virtual, posibilitando la creación de una realidad mixta o mezclada. Entre estas modalidades la permeabilidad entre elementos reales y virtuales es omnipresente y proporciona múltiples posibilidades educativas.

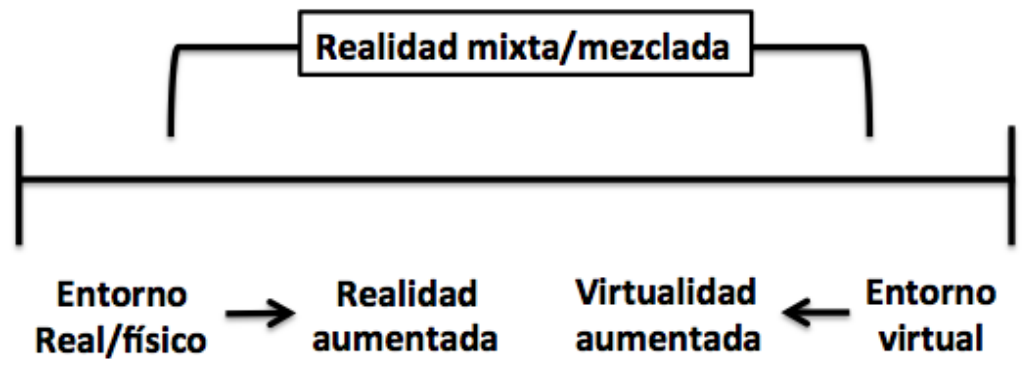

Figura 2. Continuum Realidad-Virtualidad. Adaptado de Drgoldie

http://commons.wikimedia.org/wiki/File\%3AMilgram_Continuum.png, vía Wikimedia Commons

En este grupo de nuevas (y otras no tan recientes) formas de virtualidad podemos contemplar diferentes experiencias y tecnologías que ofrecen grandes posibilidades educativas, como (de Benito, Darder \& Salinas 2014):

- La realidad virtual a través de redes, mediante el uso de juegos multiusuario y mundos virtuales.

- Tele-presencia y Tele-investigación, como formas de virtualidad aumentada.

Además, se podrían añadir: 
- La realidad aumentada, como tecnología que permite enriquecer aspectos reales utilizando elementos virtuales.

- El U-Learning o aprendizaje ubicuo como ambiente de aprendizaje permeable y mixto, en que no hay distinción entre lo real y lo virtual.

En el lado del continuum más cercano a la virtualidad, encontramos los juegos multiusuario y mundos virtuales como entornos virtuales y la virtualidad aumentada.

Los juegos multiusuario a que se ha hecho referencia anteriormente, concebidos especialmente para ser utilizados a través de una red telemática, se están transformando en una de las aplicaciones más sofisticadas de la red dirigidas al ocio. Una de las características fundamentales de estos juegos es su capacidad de crear una comunidad de jugadores que opera simultáneamente sobre un espacio simbólico colectivo. La existencia de 'mundos tridimensionales', la manipulación de objetos dentro de estos mundos, constituyen un fuerte atractivo. Los videojuegos más sofisticados pueden ser considerados casos de televirtualidad.

Los mundos virtuales han sido una de las tecnologías virtuales y "videojuegos" más sofisticados con más éxito en el campo educativo. Estas tecnologías posibilitan la creación de entornos virtuales en los cuales los usuarios pueden adoptar un avatar (sus representaciones digitales) e interaccionar con otros usuarios. SecondLife u OpenSim (versión libre de SecondLife) son dos ejemplos de tecnologías de este tipo. El uso de estos entornos en la educación se basa en el aprendizaje por descubrimiento, pues el alumno aprende a través de la interacción con los objetos y otros usuarios de ese mundo virtual. El alumno puede construir el mundo en el que desea interactuar, armando paisajes y fabricando objetos digitales (de Oliveira, Gallardo, Bettencourt \& Gisbert, 2012).

La posibilidades educativas que ofrecen son amplias. A modo de ejemplo, podemos indicar: el desarrollo de clases virtuales, conferencias, debates, el acceso a recursos de aprendizaje en 3D, simulaciones, aprender de forma colaborativa y en comunidad. Por tanto, son entornos que permiten el diseño y la manipulación del ambiente de aprendizaje y la comunicación en tiempo real entre los alumnos, llegando a simular situaciones de la vida bastante realistas. Por todo ello, se trata de entornos virtuales que permiten un nivel de "presencialidad" muy alto, facilitando la interacción y la comunicación entre los participantes, aunque se traten de entornos virtuales no reales.

En este contexto, comienzan a desarrollarse experiencias educativas que incorporan este tipo de juegos a los procesos de enseñanza-aprendizaje, como son los llamados "Serious games". Estos fueron inicialmente concebidos para entrenar a trabajadores para tareas en trabajos específicos (por ejemplo, al ejército o personal de ventas). Permiten aprovechar las posibilidades de las tecnologías para la simulación y visualización, y de esta manera contextualizar la experiencia del jugador en entornos realistas que suponen verdaderos retos, aportan libertad para la exploración de diversas situaciones $\mathrm{y}$, en muchos casos, también favorecen la colaboración (de Gloria, Bellotti, Berta \& Lavagnino, 2014).

La tele-presencia, tele-investigación o acceso remoto permite experimentar visual y auditivamente lo que sucede en otro lugar. En este caso, se trata de aplicaciones que permiten la exploración de lugares y objetos reales a través de juegos, laboratorios virtuales, etc. en tiempo real y sin necesidad de desplazarse. 
También posibilitan, al igual que los mundos virtuales, la manipulación de objetos, la creación de entornos virtuales y la interacción en lugares remotos o con seres virtuales (de Benito, Darder \& Salinas 2014). Los laboratorios virtuales permiten a los alumnos realizar prácticas que no podrían hacer en condiciones reales por diferentes motivos, por las características de la tarea, el acceso a la actividad o al campus.

En el lado del continuum más cercano a la realidad, encontramos la realidad aumentada y el u-learning.

La realidad aumentada consiste en "añadir información virtual sobre la realidad; este proceso se realiza en tiempo real en función de lo que captura una cámara de un dispositivo y se establece, además, una relación espacial entre la información virtual y su entorno real" (Estebanell, Ferrés, Cornellà \& Codina, 2012, p.136). Por tanto, se trata de que lo que ve el alumno en el mundo real se complementa con información virtual superpuesta al real, pudiendo interactuar con ella sin perder el contacto con el mundo real (Basogain, Olabe, Espinosa, Rouèche \& Olabe, 2007). Hay principalmente dos tipos de realidad aumentada: la basada en el reconocimiento de formas (a través de marcadores, imágenes u objetos) y la basada en el reconocimiento de la posición.

El U-Learning o Aprendizaje ubicuo se hace efectivo debido a la posibilidad de acceder a la información a través de tecnología móvil (smartphones, tabletas, PDAs,...) en cualquier lugar y momento, por lo que el aprendizaje también puede producirse independientemente del contexto físico y temporal (Salinas, 2012; Salinas, Darder \& de Benito, 2014). En la clase ubicua, los alumnos se mueven en torno a un espacio ubicuo e interaccionan con varios dispositivos. Se trata de una situación en la que el aprendizaje es omnipresente, por lo que siempre está sucediendo, aunque el alumno pueda no ser consciente del proceso de aprendizaje (Jones \& Jo, 2004).

\section{La necesidad de repensar la didáctica universitaria (rediseños de acciones formativas)}

Es en este contexto de nuevos escenarios de aprendizaje, derivados en gran parte del impacto de lo virtual sobre la didáctica universitaria, donde cabe la necesidad de replantear cómo se está acometiendo los nuevos procesos didácticos y cómo avanzar hacia la incorporación real de cambio de metodologías y los enfoques de aprendizaje centrado en el alumno.

La tecnología disponible y sus posibilidades educativas, especialmente haciendo referencia al aprendizaje ubicuo desde el momento en que cada persona puede aprender en cualquier lugar y momento, hacen que las fronteras entre lo formal y lo informal resulten cada vez menos nítidas y se entrecrucen.

Ese cambio de metodologías, de transmisivas y centradas en el profesor, a activas y centradas en el alumno, debe orientarse a la gestión y construcción del conocimiento personal del alumno y, para ello, será importante promover y fortalecer su entorno personal de aprendizaje (PLE), como la intersección de todos los elementos (fuentes de información, relaciones y conexiones, herramientas, estrategias) tanto de aprendizaje formal como informal (Salinas, 2013).

La universidad como institución educativa, tiene un importante papel en el desarrollo del PLE del alumno pues, como indican Rodríguez Ilera, Rubio, Galván \& Barberà (2014, p.6), "la configuración y uso de los PLE depende en gran parte de que el docente planifique actividades de aprendizaje centradas en el estudiante como 
situaciones en las que se produzca un efecto con en el uso del espacio personal y sus herramientas y tareas".

La evolución de la virtualización incorpora cambios a la docencia universitaria (incorporación de lo social, el fortalecimiento de los entornos personales como forma de promover la gestión y construcción del conocimiento personal, etc.) que hacen evolucionar la forma en que los procesos didácticos se realizan en los entornos cada vez más virtuales, orientando el foco de interés hacia nuevas metodologías de enseñanza-aprendizaje en la educación superior.

\section{Referencias}

Basogain, X., Olabe, M., Espinosa, K., Rouèche, C., \& Olabe, J. C. (2007). Realidad Aumentada en la Educación: una tecnología emergente. En $7^{a}$ Conferencia Internacional de la Educación y la Formación basada en las Tecnologías. Madrid.

Batista, G., Borges, F., \& Forés, A. (2006). Didáctica universitaria en Entornos Virtuales de Enseñanza-Aprendizaje. Madrid: Narcea.

de Benito, B., Darder, A., \& Salinas, J. (2014). Audio y video digital en la práctica educativa. En J. Cabero \& J. Barroso (Eds.), Tecnología Educativa. Más allá de hablar de TIC aplicadas a la Educación. Madrid: Síntesis.

de Gloria, A., Bellotti, F., Berta, R., \& Lavagnino, E. (2014). Serious Games for education and training. International Journal of Serious Games, 1(1). doi: 10.17083/ijsg.v1i1.11

Estebanell, M., Ferrés, J., Cornellà, P., \& Codina, D. (2012). Realidad aumentada y códigos QR en educación. En Tendencias emergentes en educación con TIC, pp. 135157. Espiral.

Jones, V., \& Jo, J. H. (2004, December). Ubiquitous learning environment: An adaptive teaching system using ubiquitous technology. In Beyond the comfort zone: Proceedings of the 21st ASCILITE Conference (No. 468, p. 474). 
de Oliviera, J., Gallardo, E., Bettencourt, T. \& Gisbert, M. (2012). Meandros de la interacción: Desafíos del uso pedagógico de los entornos virtuales 3D. Revista Iberoamericana, 60, Sept-Dic 2012. Disponible en http://rieoei.org/rie_revista.php?numero=rie60a10\&titulo=Lo\%2520narrativo\%2520y \%2520visual\%2520de\%2520Voces\%2520en\%2520el\%2520parque:\%2520una\%2520 propuesta\%2520did\%25C3\%25A1ctica\%2520interdisciplinar\%2520en\%2520el\%252 0aula\%2520de\%2520secundaria

Mishra, P. \& Koehler, M.J. (2008). Introducing TPCK. En Handbook of Technological Pedagogical Content Knowledge (TPCK) for Educators. Disponible en http://www.mendeley.com/research/what-is-technological-pedagogical-contentknowledge-tpack/

Rodríguez Ilera, J. L., Rubio, M. J., Galván, C., \& Barberà, E. (2014). Diseño de un entorno mixto e-portfolio/ple centrado en el desarrollo de competencias transversales. EDUTEC, Revista Electrónica de Tecnología Educativa, (47).

Salinas, J. (2002). Modelos flexibles como respuesta de las universidades a la sociedad de la información. Acción Pedagógica, 11(1), 4-13. Disponible en http://formamente.guideassociation.org/wpcontent/uploads/2013/10/2006_0_0_JesusSalinas.pdf

Salinas, J. (2012). La investigación ante los desafíos de los escenarios de aprendizaje futuros. RED, 32. Disponible en http://revistas.um.es/red/article/view/233091

Salinas, J. (2013). Enseñanza Flexible y Aprendizaje Abierto, Fundamentos clave de los PLEs. In L. Castañeda \& J. Adell (Eds.), Entornos personales de aprendizaje: Claves para el ecosistema educativo en red (pp. 53-70). Alcoy: Marfil.

Salinas, J., de Benito, B., \& Lizana, A. (2014). Competencias docentes para los nuevos escenarios de aprendizaje. Revista Interuniversitaria de Formación del Profesorado, $\quad 79 \quad$ (28.1), $145-163 . \quad$ Disponible en http://www.redalyc.org/articulo.oa?id=27431190010

Salinas, J., Darder, A., \& de Benito, B. (2014). Las TIC en la enseñanza superior: elearning, b-learning y m-learning. En J. Cabero \& J. Barroso (Eds). Tecnología Educativa. Más allá de hablar de TIC aplicadas a la Educación. Madrid: Síntesis. 
Shulman, L. (1986). Those who understand: Knowledge growth in teaching. Educational Researcher, $15 \quad$ (2), 4-14. Disponible en http://www.jstor.org/stable/1175860

Zabalza, M. (2008). La Didáctica universitaria. Bordón, 59, 2-3, pp. 489-509.

Zabalza, M. (2011). Nuevos enfoques para la didáctica universitaria actual. PERSPECTIVA, v. 29(2), 387-416. doi: 10.5007/2175-795X.2011v29n2p387 